\title{
Omicron variant of the SARS-CoV-2: a quest to define the consequences of its high mutational load
}

\author{
Jorge Quarleri (1) - Veronica Galvan • \\ M. Victoria Delpino
}

Received: 9 December 2021 / Accepted: 13 December 2021 / Published online: 18 December 2021

(C) The Author(s), under exclusive licence to American Aging Association 2021

The most recent wave of infections has been closely related to the emergence of the Delta variant of SARS-CoV-2. Like other RNA viruses, this virus constantly mutates with new variants emerging as long as ongoing transmission persists [5]. A variant can become more common if it provides a selective advantage to the virus.

The Technical Advisory Group on SARS-CoV-2 Virus Evolution (TAG-VE) is an independent group of experts from WHO that periodically monitors and evaluates the evolution of SARS-CoV-2 and assesses if specific mutations and combinations of mutations alter the behavior of the virus. On 26 November

J. Quarleri $(\bowtie) \cdot$ M. V. Delpino

Instituto de Investigaciones Biomédicas en Retrovirus y Sida (INBIRS), Facultad de Medicina, Consejo de Investigaciones Científicas y Técnicas (CONICET), Universidad de Buenos Aires, Buenos Aires, Argentina e-mail: quarleri@fmed.uba.ar

V. Galvan

Department of Biochemistry and Molecular Biology, University of Oklahoma Health Sciences Center, Oklahoma City, OK, USA

V. Galvan

Center for Geroscience and Healthy Brain Aging, University of Oklahoma Health Sciences Center, Oklahoma City, OK, USA

V. Galvan

Research Health Scientist, US Department of Veterans Affairs, Oklahoma City VA Health Care System, Oklahoma City, OK, USA
2021, this group was organized to analyze the emergence of the new SARS-CoV-2 variant classified as B.1.1.529, which was first reported to WHO from the Network for Genomics Surveillance in South Africa on 24 November 2021. There, infections have enlarged abruptly from November 9, when the first known B.1.1.529 infection was confirmed. Up to now, the variant has now been detected in South Africa, Botswana, the UK, the Netherlands, Italy, Germany, Spain, Belgium, Canada, Japan, Australia, Israel, Brazil, and China, among other countries. The magnitude of the actual spread of the Omicron variant around the world, however, remains uncertain as countries register new cases each day. The USA has yet to identify any cases, but there is relative certainty that this variant may already be circulating in the USA.

Based on evidence indicative of a detrimental change in COVID-19 epidemiology, the Technical Advisory Group on SARS-CoV-2 Virus Evolution (TAG-VE) has advised the World Health Organization (WHO) that this variant should be designated as a Variant of Concern (VOC), and the WHO has designated B.1.1.529 as a VOC, named Omicron [11]. Omicron (lineage B.1.1.529) joins to the four variants of the SARS-CoV-2 already designated as VOC including Alpha (lineage B.1.1.7, the so-called UK variant), Beta (lineage B.1.351, the so-called South Africa variant), Gamma (lineage P.1, the so-called Brazil variant), and Delta (lineage B.1.617.2).

The new Omicron variant appears to rapidly have replaced other variants in South Africa but this 
phenomenon may be biased considering that virus characterization has increased with the number of cases and remains largely focused on the area of the emergence of the Omicron variant. The occurrence of a series of super spreading events could not be discarded. A comparison of different Omicron genomes (carried out by Dr. Kristian Andersen at the Scripps Research Institute, La Jolla, CA, USA) estimates the virus emerged sometime around late September or early October 2021, which suggests it might be spreading more slowly than it appears [4].

Omicron has an exceptional number of mutations in the spike (S) protein, the viral ligand that recognizes host cells interacting with the human angiotensin-converting enzyme 2 (ACE2) receptor and is the main target of the body's immune responses. The mutations identified in the Omicron variant include several ones that have been previously identified and have been deemed of concern due to their potential impact on the course of the pandemic. The first genome-sequencing data obtained from Botswana showed 50 mutations overall in this new variant with 32 changes to the $\mathrm{S}$ protein. Many of the changes have been found in Delta and Alpha variants. These mutations are linked to heightened infectivity and the ability to evade infection-blocking antibodies.

The receptor-binding domain (RBD) is the portion of the S protein that binds directly to human ACE2 receptors. There are several amino acid changes such as K417N, N440K, G446S, S477N, T478K, E484A, Q493K, G496S, Q498R, N501Y, and Y505H at the $\mathrm{RBD}$ of the $\mathrm{S}$ protein of the Omicron variant. Omicron shares 2 out of 3 RBD mutations with the Delta variant. The first, a lysine to asparagine substitution at position 417 (not present among all Delta sequences but frequent in the Beta variant), has been associated with conformational changes to $S$ protein, which may aid in immune escape. The second mutation, a threonine to lysine substitution at position 478 , has been expected to increase the electrostatic potential and steric interference of the residue, which may further increase RBD binding affinity and enable immune escape. The third RBD mutation, present in Delta but not in the Omicron variant, is a leucine to arginine substitution at position 452, known to increase affinity for ACE2 receptors found on the surface of a variety of human cells, including the lungs [10].

The full profile of amino acid changes in the $\mathrm{S}$ protein includes $\mathrm{A} 67 \mathrm{~V}, \Delta 69-70, \mathrm{~T} 95 \mathrm{I}, \mathrm{G} 142 \mathrm{D} /$
$\Delta$ 143-145, $\Delta$ 211/L212I, ins214EPE (3 amino-acid insertion), G339D, S371L, S373P, S375F, K417N, N440K, G446S, S477N, T478K, E484A, Q493K, G496S, Q498R, N501Y, Y505H, T547K, D614G, H655Y, N679K, P681H, N764K, D796Y, N856K, Q954H, N969K, and L981F. The P681H mutation has also been reported in other VOCs such as Alpha, in some Gamma, and also in variants of interest (VOI) such as $\mathrm{Mu}$ and B.1.1.318. The N679K, N501Y, P681H, N679K, and D614G mutations have been related to high transmissibility [3]. The combination of mutations Q498R and N501Y in in vitro evolution studies significantly increased the binding affinity to ACE2 [14].

For cellular entry, the SARS-CoV-2 spike protein must be cleaved twice by host proteases that hydrolyze peptide bonds at specific amino acid sequences. One of these sites is a furin cleavage site required to produce pre-activated viral particles during replication and before budding from an infected cell. The Delta $S$ protein includes a mutation (P681R) located at a furin cleavage site that separates the spike 1 (S1) and S2 subunits that enhances SARSCoV-2 fitness as compared to the Alpha variant. A particular cluster of mutations present at the S1-S2 furin cleavage site in the Omicron variant (H655Y, N679K, P681H) may also be associated with increased transmissibility [2]. Whether this large number of mutations may make the virus unstable, however, needs to be defined.

Current SARS-CoV-2 PCR diagnostics are effective for the detection of the Omicron variant. A deletion at positions $69-70$ of the $S$ protein $(\Delta 69-70)$ has been observed that was also found in Alpha and Eta variants among others. This $\Delta 69-70$ deletion leads to false-negative readings in the S-assay of the widely used PCR test (TaqPath, ThermoFisher, USA). This particularity (a phenomenon referred to as S-gene target failure or SGTF, or S gene dropout) may provide a useful proxy for measures of prevalence of Omicron. The PCR test can therefore be used to identify the Omicron variant pending sequencing confirmation. However, because the $\Delta 69-70$ deletion is also present in other variants, confirmation studies must be performed to rule out the presence of other variants. Studies are ongoing to determine whether there is any impact of extensive sequence changes in Omicron on other types of diagnostic tests, including rapid antigen detection tests. 
In addition to the mutations described above, a 3 amino-acid deletion in ORF1a at L3674-, S3675-, and G3676- (also annotated as a deletion in NSP6 from 105-107) is present in the Omicron variant. There is some speculation that this mutation could aid in innate immune evasion, possibly by compromising the ability of infected cells to degrade viral components $[1,8]$.

Finally, two additional mutations in nucleocapsid (N) protein R203K and G204R are present in the Omicron variant [3]. Although these are ancestral mutations (not new to this variant), these changes are linked to increased subgenomic RNA expression [6] and increased viral loads [7].

The central uncertainties at this time are (1) how transmissible the Omicron variant is and whether any increases in transmission are related to immune escape, intrinsic increased transmissibility, or both; (2) how well vaccines protect against Omicron infection, transmission, clinical disease of different degrees of severity, and death; and (3) does the variant present with a different severity profile $[12,13]$.

It is not yet clear whether Omicron is more transmissible (e.g., more easily spread from person to person) as compared to other variants, including Delta. There was an uptick of people testing positive in Gauteng Province, South Africa, affected by this variant, but epidemiological studies that will define whether this increase is related to Omicron, or other factors, are still ongoing. Likewise, whether Omicron causes more severe disease compared to infections with other variants, including Delta, is still unknown. Preliminary data suggest increasing rates of hospitalization in South Africa, but this may be due to an increase in overall numbers of people becoming infected with different variants, rather than a result of infection with Omicron. There is currently no information to suggest that symptoms associated with Omicron are different from those from other variants. Initially reported infections were among younger individuals (university students) who tend to have milder disease. Understanding of overall severity of disease caused by the Omicron variant will take many days, likely several weeks. A report from the African Medical Association states that Omicron is seven times more contagious than the Delta variant, but the reported cases and deaths in Africa have continued to decline, and the people infected by Omicron did not show any serious aggravation in their condition. All variants of COVID-19, including the Delta variant that is dominant worldwide, can cause severe disease or death, in particular for the most vulnerable individuals including but not limited to older adults. Preventive that use all tools currently available measures (masking, distancing, vaccination) should be implemented immediately, urgently so in the case of vulnerable populations.

The high number of mutations present in the $\mathrm{S}$ protein in the Omicron variant may increase the virus's ability to evade infection-blocking antibodies, as well as other immune responses such as the $\mathrm{T}$ cell response. This observation is in agreement with preliminary evidence suggesting an increased risk of reinfection with Omicron as compared to other VOC, but the information is still scarce. A retrospective analysis of suspected reinfections among South African individuals offers evidence of a temporally juxtaposed Omicron emergence with a higher risk of reinfection, not observed during previous waves associated with Beta, or Delta variants, suggesting that Omicron variant is associated with a particular ability to escape natural immunity from prior infection [9]. Although herd immunity became a remote goal after the emergence of the Delta variant, it is still premature to say if Omicron will be a greater challenge to achieve that goal.

The impact of Omicron on the effectiveness of vaccines is currently under evaluation. Breakthrough infections with Omicron have been reported in South Africa among people who have received any of the three kinds of vaccines in use there (Ad26.COV2.S from Janssen -Johnson \& Johnson-, BNT162b2 from Pfizer-BioNTech, and AZD1222 Oxford/AstraZeneca). According to recent news reports, two quarantined travelers in Hong Kong who tested positive for the Omicron variant had received 2 doses of the Pfizer vaccine. One individual had traveled from South Africa; the other was infected during hotel quarantining [13]. Ideally, the administration of a vaccine that targets the spike protein as a booster will allow the expansion of memory B lymphocytes producing neutralizing antibodies. Despite the presence of numerous mutations in the S1 region of the Omicron spike, the shared regions of the S2 with the original vaccine strain are expected to induce a broadly reactive immune response. This is an important aspect of immunity that may help preserve vaccine-driven immunity in the face of infection with the Omicron variant. 
The goal of this article is to summarize information publicly available about the Omicron variant of SARS-CoV-2. Ongoing laboratory studies and soonto-be-available clinical and epidemiological data will shed light on the biological and clinical consequences of the high mutational load in the Omicron variant.

\section{Declarations}

Conflict of interest The authors declare no competing interests.

\section{References}

1. Benvenuto D, Angeletti S, Giovanetti M, Bianchi M, Pascarella S, Cauda R, Ciccozzi M, Cassone A. Evolutionary analysis of SARS-CoV-2: how mutation of Non-Structural Protein 6 (NSP6) could affect viral autophagy. J Infect. 2020;81:e24-7.

2. Gong SY, Chatterjee D, Richard J, Prevost J, Tauzin A, Gasser R, Bo Y, Vezina D, Goyette G, Gendron-Lepage G, Medjahed H, Roger M, Cote M, Finzi A. Contribution of single mutations to selected SARS-CoV-2 emerging variants spike antigenicity. Virology. 2021;563:134-45.

3. Hodcroft EB. CoVariants: SARS-CoV-2 mutations and variants of interest. $2021 \mathrm{https} / / /$ covariants.org/. Accessed 1 December 2021.

4. Kupferschmidt K. 'Patience is crucial': why we won't know for weeks how dangerous Omicron is. 2021 https://www.science.org/content/article/patience-cruci al-why-we-won-t-know-weeks-how-dangerous-omicron. Accessed 27 November 2021.

5. Lauring, A.S. and Malani, P.N., 2021, Variants of SARSCoV-2. JAMA.

6. Leary, S., Gaudieri, S., Parker, M.D., Chopra, A., James, I., Pakala, S., Alves, E., John, M., Lindsey, B.B., Keeley, A.J., Rowland-Jones, S.L., Swanson, M.S., Ostrov, D.A., Bubenik, J.L., Das, S., Sidney, J., Sette, A., consortium, C.-G.U., de Silva, T.I., Phillips, E. and Mallal, S., 2021, Generation of a novel SARS-CoV-2 sub-genomic RNA due to the R203K/G204R variant in nucleocapsid: homologous recombination has potential to change SARSCoV-2 at both protein and RNA level. bioRxiv.

7. Mourier, T., Shuaib, M., Hala, S., Mfarrej, S., Alofi, F., R., N., Alsomali, A., Jorgensen, D., Subudhi, A.K., Rached, F.B., Guan, Q., Salunke, R.P., Ooi, A., Esau, L., Douvropoulou, O., Nugmanova, R., Perumal, S., Zhang, H., Rajan, I., Al-Omari, A., Salih, S., Shamsan, A., Al Mutair, A., Taha, J., Alahmadi, A., Khotani, N., Alhamss,
A., Mahmoud, A., Alquthami, K., Dageeg, A., Khogeer, A., Hashem, A.M., Moraga, P., Volz, E., Almontashiri, N. and Pain, A., 2021, Saudi Arabian SARS-CoV-2 genomes implicate a mutant Nucleocapsid protein in modulating host interactions and increased viral load in COVID-19 patients. . medRxiv preprint: 1-38.

8. Pang, X., Li, P., Zhang, L., Que, L., Dong, M., Xie, B., Wang, Q., Wei, Y., Xie, X., Li, L., Yin, C., Wei, L., Huang, K., Hua, Y., Zhou, Q., Li, Y., Yu, L., Li, W., Mo, Z., Zhang, M., Leng, J. and Hu, Y., 2021, Emerging severe acute respiratory syndrome coronavirus 2 mutation hotspots associated with clinical outcomes and transmission. Front Microbiol, 12: 753823.

9. Pulliam, J.P., van Schalkwyk, C., Govender, N., von Gottberg, A., Cohen, C., Groome, M.J., Dushoff, J., Mlisana, K. and Moultrie, H., 2021, Increased risk of SARS-CoV-2 reinfection associated with emergence of the Omicron variant in South Africa. medRxiv preprint.

10. Sanches, P.R.S., Charlie-Silva, I., Braz, H.L.B., Bittar, C., Freitas Calmon, M., Rahal, P. and Cilli, E.M., 2021, Recent advances in SARS-CoV-2 Spike protein and RBD mutations comparison between new variants Alpha (B.1.1.7, United Kingdom), Beta (B.1.351, South Africa), Gamma (P.1, Brazil) and Delta (B.1.617.2, India). J Virus Erad, 7: 100054.

11. WHO, 2021a, Classification of Omicron (B.1.1.529): SARS-CoV-2 Variant of Concern. 2021. https://www. who.int/news/item/26-11-2021-classification-of-omicron(b.1.1.529)-sars-cov-2-variant-of-concern. Accessed 26 November 2021.

12. WHO, 2021b, Enhancing readiness for Omicron (B.1.1.529): technical brief and priority actions for member states. https://www.who.int/publications/m/item/enhan cing-readiness-for-omicron-(b.1.1.529)-technical-briefand-priority-actions-for-member-states. Accessed 28 November 2021.

13. WHO, 2021c, Update on Omicron. 2021. https://www. who.int/news/item/28-11-2021-update-on-omicron. Accessed 28 November 2021.

14. Zahradnik J, Marciano S, Shemesh M, Zoler E, Harari D, Chiaravalli J, Meyer B, Rudich Y, Li C, Marton I, Dym O, Elad N, Lewis MG, Andersen H, Gagne M, Seder RA, Douek DC, Schreiber G. SARS-CoV-2 variant prediction and antiviral drug design are enabled by RBD in vitro evolution. Nat Microbiol. 2021;6:1188-98.

Publisher's note Springer Nature remains neutral with regard to jurisdictional claims in published maps and institutional affiliations. 